\title{
ZEROS OF ANALYTIC FUNCTIONS WITH INFINITELY DIFFERENTIABLE BOUNDARY VALUES
}

\author{
JAMES G. CAUGHRAN ${ }^{1}$
}

\begin{abstract}
A necessary and sufficient condition is proved that a set of points $\left\{r_{n} e^{i \theta_{n}}\right\}$ in the unit disk be the set of zeros of an analytic function with infinitely differentiable boundary values for every choice of $\left\{r_{n}\right\}, 0<r_{n}<1$ and $\sum\left(1-r_{n}\right)<\infty$.
\end{abstract}

1. Introduction. The algebra $A^{\infty}$ is the class of all functions analytic in the open unit disk $\boldsymbol{D}$ with all derivatives bounded in $\boldsymbol{D}$ or, alternatively, the class of all bounded analytic functions with boundary values $f\left(e^{i \theta}\right)=\lim _{r \rightarrow 1} f\left(r e^{i \theta}\right)$ having infinitely many continuous derivatives. Beurling [1, p. 13], Carleson [2], and Novinger [5] have characterized the boundary zeros of such functions, while Taylor and Williams [6] have discovered several further properties of this class relating to zeros. Little, however, is known about the zeros within $D$ beyond a few partial results (see [4] and [8]).

In this paper, an apparently unrelated sufficient condition on the points is presented (Theorem 2). If $z_{n}=r_{n} e^{i \theta_{n}}$ satisfy the Blaschke condition and if the closure of the set $\left\{e^{i \theta_{n}}: n=1,2, \cdots\right\}$ of projections of the points to the boundary $T$ of $D$ forms a Carleson set, then there is a nonzero function $f \in A^{\infty}$ such that $f\left(z_{n}\right)=0$. Thus the points may converge to their limit set as tangentially as desired provided they are "well spaced out."

Together with a slightly altered version of the construction in [4], Theorem 1 , this result provides a necessary and sufficient condition that $\left\{r_{n} e^{i \theta_{n}}\right\}$ be the zeros of an $A^{\infty}$ function for every choice of $\left\{r_{n}\right\}$, $0<r_{n}<1$ and $\sum\left(1-r_{n}\right)<\infty$.

The construction requires some knowledge of $A^{\infty}$ functions in other domains than the unit disk. This is discussed in $\$ 2$, where the analogue of the Carleson-Novinger result on boundary zeros is formulated in a simply-connected Jordan domain with smooth boundary. $\S 3$ is devoted to some growth estimates used in the construction. Finally, the construction forms $\S 4$ of this paper.

Presented to the Society, November 29, 1969; received by the editors June 20, 1969.

AMS Subject Classifications. Primary 3085, 3087; Secondary 4630.

Key Words and Phrases. Bounded analytic function, zero set, boundary zeros, domain with smooth boundary, Carleson set, Blaschke product.

1 This research was partially supported by NSF grants GP 8225 and GP 11725 . 
2. Boundary zeros of $A^{\infty}$ functions. Let $R$ be a domain in the complex plane, and let $\boldsymbol{A}^{\infty}(\boldsymbol{R})=\left\{f: f^{(n)}\right.$ is analytic and bounded in $R$, for $n=1,2, \cdots\}$. In this section the possible boundary zeros of functions in $A^{\infty}(R)$ are determined for a Jordan domain $R$ with a smooth boundary, i.e., $R$ is bounded by a rectifiable Jordan curve $w=w(s)$, where $w$ is infinitely differentiable with respect to arc length $s$.

Lemma 1. If $R$ is a Jordan domain with smooth boundary, then if $\psi$ is the mapping function from $R$ to the unit disk $D, \psi \in A^{\infty}(R)$ and $\phi$ $=\psi^{-1} \in A^{\infty}(D)$. Moreover, $\phi^{\prime}(z) \neq 0$ for $z \in \bar{D}$.

Proof. That $\phi \in A^{\infty}(D)$ follows from a theorem of Kellogg (see [7]): if the boundary function $w$ has $n+2$ bounded derivatives with respect to arc-length $(n \geqq 1)$, then $\phi$ has $n+1$ bounded derivatives in $D$, and thus $n$ derivatives continuously extendable to the closed disk $\bar{D}$. Warschawski also showed $\phi^{\prime}(z)$ is nowhere zero in $\bar{D}$. Thus it is possible to solve for the derivatives of $\psi$ in terms of those of $\phi$, and therefore they are bounded in $R$.

A Carleson set $E$ is a closed set of measure zero contained in the unit circle $T$ for which, if the intervals complementary to $E$ have lengths $\epsilon_{n}, \sum \epsilon_{n} \log \epsilon_{n}>-\infty$. Novinger [5] showed that every Carleson set is the set of boundary zeros of an $A^{\infty}$ function, while Beurling [1] proved that the zeros in $T$ of any function, analytic in $D$ and continuous in $\bar{D}$ which satisfies a Lipschitz condition on $T$, must form a Carleson set.

We define a Carleson set for a Jordan domain $R$ with smooth boundary $\partial R$ to be a closed set $E \subset \partial R$ of linear measure zero, whose complementary arcs satisfy the same finiteness condition. The following lemma is an easy consequence of the boundedness of the first derivatives of the mapping functions.

Lemma 2. If $\boldsymbol{R}$ is a Jordan domain with smooth boundary, $\psi$ is the mapping function from $R$ to the unit disk, and $E \subseteq \partial R$, then $E$ is a Carleson set in $\partial R$ if and only if $\psi(E)$ is a Carleson set in $T$.

THEOREM 1. Let $R$ be a Jordan domain with smooth boundary. If $f$ is analytic in $R$ and continuous in $\bar{R}$, and if $f$ satisfies a Lipschitz condition on $\partial R$, then the zeros of $f$ in $\partial R$ form a Carleson set in $\partial R$. Conversely, if $E$ is a Carleson set in $\partial R$, there is a function $f \in A^{\infty}$ which has a zero of infinite order at each point of $E\left(i . e ., f^{(j)}(z)=0, j=0,1,2, \ldots\right.$ for $z \in E)$, and no other zeros.

Proof. If $f$ is analytic in $R$ and satisfies the Lipschitz condition $\left|f\left(z_{1}\right)-f\left(z_{2}\right)\right| \leqq K\left|z_{1}-z_{2}\right|^{\alpha}$ for $z_{1}, z_{2} \in \partial R$, then by Lemma $1, f \circ \phi$ is 
analytic in $D$ and satisfies a Lipschitz condition of the same order, where $\phi$ is the mapping function from $D$ to $R$. By Beurling's proof, the zero set of $f \circ \phi$ is a Carleson set, and by Lemma 2, the boundary zero set of $f$ is a Carleson set in $\partial R$.

If $E$ is a Carleson set in $\partial R$, Novinger's construction provides a function $g \in A^{\infty}(D)$ vanishing on $\phi^{-1}(E)$ and nowhere else. By Lemma $1, f=g \circ \phi^{-1}$ is the desired function.

3. Magnitude of Blaschke products and $A^{\infty}$ functions. In this section we present some estimates on the derivatives of Blaschke products and $A^{\infty}$ functions. Similar estimates were proved by Wells [8].

If $z_{k} \in D$ and $\sum\left(1-\left|z_{k}\right|\right)<\infty$, then the Blaschke product with zeros $z_{k}$,

$$
B(z)=\prod \frac{\bar{z}_{k}}{\left|z_{k}\right|} \frac{z_{k}-z}{1-\bar{z}_{k} z},
$$

converges in $D$ to a bounded analytic function with radial limits $B\left(e^{i \theta}\right)$ of modulus 1 almost everywhere. Any bounded function $f$ analytic in $D$ has a factorization $f=F B$, where $B$ is a Blaschke product and $F$ has no zeros. Thus estimates of the growth of the derivatives of Blaschke products are essential to the construction.

Lemma 3. If $B$ is a Blaschke product with zeros $z_{k}=r_{k} e^{i \theta_{k}}$, where $r_{k}>1 / 2$, then there is a sequence of positive numbers $N_{j}$ for which, if $A$ is any subproduct of $B$,

$$
\left|A^{(j)}(z)\right| \leqq N_{j} \operatorname{dist}(z, K)^{-2 j}, \quad j=1,2, \cdots,
$$

where $K=\left\{1 / \bar{z}_{k}: k=1,2, \cdots\right\}$.

Proof. Differentiating $B$,

$$
B^{\prime}(z)=\sum_{k=1}^{\infty} B_{k}(z) \frac{r_{k}^{2}-1}{\left(1-\bar{z}_{k} z\right)^{2}},
$$

where $B_{k}(z)=B(z)\left(1-\bar{z}_{k} z\right) /\left(z_{k}-z\right)$. The modulus of $B^{\prime}$ is thus bounded by

$$
\sum_{k=1}^{\infty} \frac{1-r_{k}^{2}}{\left|1-\bar{z}_{k} z\right|^{2}} \leqq \sum_{k=1}^{\infty} \frac{8\left(1-r_{k}\right)}{\left|\bar{z}_{k}^{-1}-z\right|^{2}} \leqq 8 \operatorname{dist}(z, K)^{-2} \sum_{k=1}^{\infty}\left(1-r_{k}\right) .
$$

Since this estimate improves if a subproduct is taken in place of $B$, the lemma holds for $j=1$.

Suppose that constants $N_{j}$ have been determined for which the inequality holds for indices $j \leqq m$. Then there exist positive numbers $a_{m j}$ such that 


$$
\left|A^{(m+1)}(z)\right| \leqq \sum_{k=1}^{\infty}\left(1-r_{k}\right) \sum_{j=0}^{m} a_{m j}\left|A_{k}^{(m-j)}(z)\right|\left|1-\bar{z}_{k} z\right|^{-(j+2)}
$$

and

$$
\left|A^{(m+1)}(z)\right| \leqq \sum_{k=1}^{\infty}\left(1-r_{k}\right) \sum_{j=0}^{m} a_{m j} N_{m-j}\left|1-\bar{z}_{k} z\right|^{-(j+2)} \operatorname{dist}(z, K)^{-2(m-j)}
$$

by the inductive hypothesis. Since $\frac{1}{2} \operatorname{dist}(z, K) \leqq\left|1-\bar{z}_{z} z\right| \leqq 2$, this is bounded by

$$
\left(\sum_{k=1}^{\infty}\left(1-r_{k}\right) \sum_{j=0}^{m} a_{m j} N_{m-j} 2^{2 j+2}\right) \operatorname{dist}(z, K)^{-2(m+1)},
$$

and the estimate holds for $A^{(m+1)}$.

Lemma 4. If $f \in A^{\infty}(R)$ and $f$ has a zero of infinite order at $z_{0} \in \partial R$, and the line joining $z$ to $z_{0}$ lies in $R$, then there are positive constants $M_{j k}$ for which

$$
\left|f^{(j)}(z)\right| \leqq M_{j k}\left|z-z_{0}\right|^{k}, \quad j, k=0,1,2, \cdots .
$$

This lemma is easily proved by integrating $f^{(k+j+1)} k$ times.

4. Construction of an $A^{\infty}$ function with given zeros. In this section the required function is constructed after the construction of a curve which is fundamental to the argument.

Lemma 5. If $R_{k} e^{i \theta_{k}}$ is a sequence of points outside the unit disk with limit points in a closed set $E$ of zero Lebesgue measure in $T$, there is a rectifiable curve $r=h(\theta)$ which is $C^{\infty}$ with respect to arc-length, and for which $1<h\left(\theta_{k}\right) \leqq R_{k}$.

The proof is left to the reader.

THEOREM 2. If $\left\{e^{i i_{n}}: n=1,2, \cdots\right\}$ is a set of points on the unit circle whose closure is a Carleson set, and if $0<r_{n}<1$ and $\sum\left(1-r_{n}\right)<\infty$, there is a function $f \in A^{\infty}$ for which $f\left(r_{n} e^{i \theta_{n}}\right)=0$. Conversely, if $\left\{r_{n} e^{i \theta_{n}}\right\}$ is contained in the zero set of an $A^{\infty}$ function for every choice of $\left\{r_{n}\right\}$, $0<r_{n}<1$ and $\sum\left(1-r_{n}\right)<\infty$, then $\left\{e^{i \theta_{n}}\right\}-$ is a Carleson set.

Proof. Let $z_{n}=r_{n} e^{i \theta_{n}} \in D$, where the closure of $\left\{e^{i \theta_{n}}: n=1,2, \cdots\right\}$ is a Carleson set and $\sum\left(1-r_{n}\right)<\infty$. We may assume $r_{n}>1 / 2$, for otherwise the function resulting from ignoring the points $z_{n}$ with $r_{n} \leqq 1 / 2$ can be multiplied by the finite Blaschke product with those zeros.

By Lemma 5 , there is a $C^{\infty}$ curve $r=h(\theta)$ between the unit circle and the points $R_{n} e^{i \theta_{n}}$, where $R_{n}=\inf \left\{1 / r_{k}: \theta_{k}=\theta_{n}\right\}$, and $h(\theta)>1$ unless $e^{i \theta}$ is a limit point of $\left\{z_{n}\right\}$. This curve will form the boundary of a 
Jordan domain $R$. Let $K=\left\{1 / \bar{z}_{n}: n=1,2, \cdots\right\}$, and let $E$ be the closure of the set of points $h\left(\theta_{n}\right) e^{i \theta_{n}}$. It is clear that $E$ is a Carleson set in $\partial R$. By Theorem 1, there is a function $F \in A^{\infty}(R)$ which has a zero of infinite order at each point of $E$. Further, the restriction of $F$ to $D$ is in $A^{\infty}(D)$.

Let $B$ be the Blaschke product with zeros $z_{n}$, and let $f(z)=F(z) B(z)$ for $z \in \bar{D}$. By Lemma $4,\left|f^{(j)}(z)\right| \leqq M_{j k} \operatorname{dist}(z, E)^{k} \leqq M_{j k} \operatorname{dist}(z, K)^{k}$ for some constants $M_{j k}$ and $j, k=0,1, \ldots$. By Lemma 3 there are constants $N_{j}$ for which $\left|B^{(j)}(z)\right| \leqq N_{j} \operatorname{dist}(z, K)^{-2 j}$. If $z \in D$,

$$
\left|f^{(j)}(z)\right|=\left|\sum_{i=0}^{j}\left(\begin{array}{l}
j \\
i
\end{array}\right) F^{(j-i)}(z) B^{(i)}(z)\right| \leqq \sum_{i=0}^{j}\left(\begin{array}{l}
j \\
i
\end{array}\right) M_{j-i, 2 i} N_{i}
$$

so $f^{(j)}$ is bounded, $j=1,2, \cdots$.

Conversely, if $\left\{e^{i \theta_{n}}\right\}-$ is not a Carleson set, a slight modification of the construction in Theorem 1 of [4] yields a sequence $\left\{r_{n}\right\}$ for which $\left\{r_{n} e^{i \theta_{n}}\right\}$ is not contained in the zero set of any function with finite Dirichlet integral, and thus not of any $A^{\infty}$ function.

Necessary conditions are difficult to find. Carleson's formula for the Dirichlet integral [3] yields the condition

$$
\int_{0}^{2 \pi} \log \sum \frac{1-\left|z_{n}\right|^{2}}{\left|e^{i t}-z_{n}\right|^{2}} d t<\infty
$$

which he used to create the counterexample in [4]. This, the Blaschke condition, and the requirement that the limit points lie in a Carleson set seem to be all that is known. The distance from these conditions to the known sufficient conditions is rather great.

\section{REFERENCES}

1. A. Beurling, Ensembles exceptionnels, Acta Math. 72 (1939), 1-13. MR 1, 226.

2. L. Carleson, Sets of uniqueness for functions regular in the unit circle, Acta Math. 87 (1952), 325-345. MR 14, 261.

3. —_ A representation formula for the Dirichlet integral, Math. Z. 73 (1960), 190-196. MR 22 \#3803.

4. J. G. Caughran, Two results concerning the zeros of functions with finite Dirichlet integral, Canad. J. Math. 21 (1969), 312-316.

5. W. P. Novinger, Holomorphic functions with infinitely differentiable boundary values, (to appear).

6. B. A. Taylor and D. L. Williams, Ideals in rings of analytic functions with smooth boundary values, (to appear).

7. S. Warschawski, Über einen Satz von O. D. Kellogg, Gött. Nach. (1932), 73-86.

8. J. H. Wells, On the zeros of functions with derizatives in $H^{1}$ and $H^{\infty}$, Canad. J. Math. (to appear).

UNIVERSITY OF KENTUCKY 